\title{
Ginseng: potential for the antileishmanial arsenal?
}

\section{Ginseng: potencial para o arsenal "leishmanicida"?}

\author{
Nader Pazyar', Reza Yaghoobi" \\ Department of Dermatology, Jundishapur University of Medical Sciences, Ahvaz, Iran
}

'MD. Assistant Professor, Department of Dermatology, Ahvaz Jundishapur University of Medical Sciences, Ahvaz, Iran.

"MD. Professor, Department of Dermatology, Ahvaz Jundishapur University of Medical

Sciences, Ahvaz, Iran.
Cutaneous leishmaniasis (CL) develops after inoculation of the skin with parasites of the genus Leishmania, transmitted by phlebotomines (sandflies). Every year, CL affects approximately 1-1.5 million people worldwide, with over $90 \%$ of the cases occurring in the Middle East and South America. ${ }^{1}$

CL is regarded as a parasitic disease caused by Leishmania and no optimal medication protocol is available for this skin infection. Beneficial treatment regimens for CL should be based on experience, efficiency and the toxicity of the drugs in each region. ${ }^{2}$

Importantly, it has been clarified that protective immunity against $\mathrm{CL}$ is related to induction of Th1/Tc1 immune response, which leads to efficient parasite elimination. ${ }^{3}$ Interleukin (IL)-12 is unanimously believed to be a pivotal cytokine for induction of Th1/Tc1-dependent protection against Leishmania. ${ }^{4}$ Moreover, activated macrophages are the major source of IL-12, which stimulates autocrine macrophage activation. ${ }^{5}$

One important point needing attention is that the polyamine biosynthetic pathway is fundamental for the growth and survival of Leishmania. Ornithine decarboxylase (ODC) is the first enzyme in the polyamine biosynthetic pathway. ${ }^{6}$ Additionally, it has been recognized that ODC catalyzes the conversion of ornithine to putrescine ${ }^{7}$ and that the levels of putrescine are elevated in cases of resistant leishmaniasis. ${ }^{8}$

It has been revealed that P-glycoproteins mediate drug resistance to Leishmania and other protozoa and that this is followed by development of cross-resistance to numerous structurally and functionally unrelated drugs. ${ }^{9}$

Leishmania parasites are obligate intracellular organisms in mammals, and they invade macrophages and dendritic cells. Notably, it has been demonstrated that nitric oxides produced in macrophages possess a crucial role as leishmanicidal agents. ${ }^{10}$

Ginseng (Panax ginseng) has been used for thousands of years in phytomedicine and it has captured a specific position on the list of the best-selling herbal agents in the world. ${ }^{11}$ Ginseng modulates blood pressure, metabolism and immune functions. ${ }^{12}$

Interestingly, ginsenosides, which are the major active component of ginseng, have a range of biomedical effects. ${ }^{13}$ They are triterpene saponins, and most ginsenosides are composed of a dammarane skeleton (17 carbons in a four-ring structure) with various sugar moieties. ${ }^{12}$

It is noteworthy that ginseng therapy has been shown to stimulate a Th1-like immune response, which improves the course of diseases in animal models. ${ }^{14}$ Importantly, ginseng modulates the peripheral blood mononuclear cells and leads to higher IL-12 production. Additionally, elevated IL-12 levels can induce a more potent Th1 immune response. ${ }^{15}$

IH-901, a new intestinal bacterial metabolite extracted from protopanaxadiol-type ginsenosides, has been observed to suppress ornithine decarboxylase activity dose-dependently in animal skin. ${ }^{16}$

Protein-arginine N-methyltransferase (protein methylase I) catalyzes methylation of arginyl residues on substrate protein post-translationally. It has been found that Korean red ginseng is able to inhibit protein methylase I activity and, subsequently, polyamines in vitro. ${ }^{17}$

Lee et al. demonstrated that total saponin in ginseng and ginsenosides was capable of decreasing the putrescine levels in immobilization-stressed gerbil mice. ${ }^{18}$ 
Notably, an in vitro study has shown that purified Rg1 ginsenosides increase the production of nitric oxide from IFNgamma activated macrophages. ${ }^{19}$

Ginsenosides have been reported to be inhibitors of P-glycoprotein (Pgp). ${ }^{20}$ Choi et al. explained that protopanaxatriol ginsenosides exert a chemosensitizing effect on Pgpmediated multidrug resistance cells. Correspondingly, this component increases the intracellular accumulation of drugs through direct interaction with Pgp at the azidopine site. ${ }^{21}$

In summary, putting the above facts together, ginseng and ginsenosides may open up a novel therapeutic opportunity for treating cutaneous leishmaniasis. Combination of topical ginseng or ginsenosides with meglumine antimoniate might boost the therapeutic effects of this drug, increase its intracellular accumulation and, subsequently, help to reduce the resistance of parasites against it.

\section{REFERENCES}

1. Wise ES, Armstrong MS, Watson J, Lockwood DN. Monitoring toxicity associated with parenteral sodium stibogluconate in the day-case management of returned travellers with New World cutaneous leishmaniasis [corrected]. PLoS Negl Trop Dis. 2012;6(6):e1688.

2. Kumaresan M, Kumar P. Localized cutaneous leishmaniasis in South India: successful treatment with ketoconazole. Indian J Dermatol Venereol Leprol. 2007;73(5):361-2.

3. von Stebut E, Udey MC. Requirements for Th1-dependent immunity against infection with Leishmania major. Microbes Infect. 2004;6(12):1102-9.

4. Zahn S, Kirschsiefen P, Jonuleit H, Steinbrink K, Von Stebut E. Human primary dendritic cell subsets differ in their IL-12 release in response to Leishmania major infection. Exp Dermatol. 2010;19(10):924-6.

5. Ota H, Takashima $Y$, Matsumoto $Y$, Hayashi $Y$, Matsumoto $Y$. Pretreatment of macrophages with the combination of IFN-gamma and IL-12 induces resistance to Leishmania major at the early phase of infection. J Vet Med Sci. 2008;70(6):589-93.

6. Singh S, Mukherjee A, Khomutov AR, et al. Antileishmanial effect of 3-aminooxy-1-aminopropane is due to polyamine depletion. Antimicrob Agents Chemother. 2007;51(2):528-34.

7. Tang X, Kim AL, Feith DJ, et al. Ornithine decarboxylase is a target for chemoprevention of basal and squamous cell carcinomas in Ptch1+/- mice. J Clin Invest. 2004;113(6):867-75.

8. Namazi MR. Hypothesis: the potential utility of topical eflornithine against cutaneous leishmaniasis. Indian J Dermatol Venereol Leprol. 2008;74(2):158-9.

9. Namazi MR. Potential utility of disulfiram against leishmaniasis. Indian J Med Res. 2008;127(2):193-4.

10. Souza AS, Giudice A, Pereira JM, et al. Resistance of Leishmania (Viannia) braziliensis to nitric oxide: correlation with antimony therapy and TNF-alpha production. BMC Infect Dis. 2010;10:209.
11. Pazyar N, Yaghoobi R. A rationale of ginseng as a novel addition to the antihirsutism armamentarium. J Altern Complement Med. 2012;18(3):210-1.

12. Leung KW, Wong AS. Pharmacology of ginsenosides: a literature review. Chin Med. 2010;5:20.

13. Pazyar N, Omidian M, Jamshydian N. Ginseng as a potential novel addition to the antikeloid weaponry. Phytother Res. 2012;26(10):1579-80.

14. Song Z, Moser $\mathrm{C}, \mathrm{Wu} \mathrm{H}$, et al. Ginseng modulerer immunresponset via påvirkning af cytokinproduktionen--sekundaerpublikation [Ginseng modulates the immune response via its effect on cytokine production--secondary publication]. Ugeskr Laeger. 2005;167(33):3054-6.

15. Larsen MW, Moser C, Høiby N, Song Z, Kharazmi A. Ginseng modulates the immune response by induction of interleukin-12 production. APMIS. 2004;112(6):369-73.

16. Lee JY, Shin JW, Chun KS, et al. Antitumor promotional effects of a novel intestinal bacterial metabolite $(\mathrm{IH}-901)$ derived from the protopanaxadiol-type ginsenosides in mouse skin. Carcinogenesis. 2005;26(2):359-67.

17. Yoo BC, Park GH, Okuda $H$, et al. Inhibitory effect of argininederivatives from ginseng extract and basic amino acids on proteinarginine N-methyltransferase. Amino Acids. 1999;17(4):391-400.

18. Lee SH, Jung BH, Kim SY, Lee EH, Chung BC. The antistress effect of ginseng total saponin and ginsenoside $\mathrm{Rg} 3$ and $\mathrm{Rb} 1$ evaluated by brain polyamine level under immobilization stress. Pharmacol Res. 2006;54(1):46-9

19. Fan ZH, Isobe K, Kiuchi K, Nakashima I. Enhancement of nitric oxide production from activated macrophages by a purified form of ginsenoside (Rg1). Am J Chin Med. 1995;23(3-4):279-87.

20. Zhou S, Lim LY, Chowbay B. Herbal modulation of P-glycoprotein. Drug Metab Rev. 2004;36(1):57-104.

21. Choi CH, Kang G, Min YD. Reversal of P-glycoprotein-mediated multidrug resistance by protopanaxatriol ginsenosides from Korean red ginseng. Planta Med. 2003;69(3):235-40.

\section{Sources of funding: None}

Conflict of interest: None

Date of first submission: May 8, 2012

Last received: February 8, 2013

Accepted: March 19, 2013

\section{Address for correspondence:}

Nader Pazyar

Department of Dermatology

Imam Hospital

Azadegan Street

Ahvaz, Iran

E-mail:dr.pazyar@gmail.com 